\title{
Co-operative Spring Migration Study 1958
}

Compiled by Mary Houston, Yorkton.

\begin{tabular}{|c|c|c|c|c|c|c|c|c|c|}
\hline & 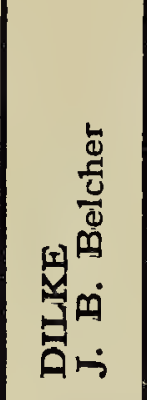 & 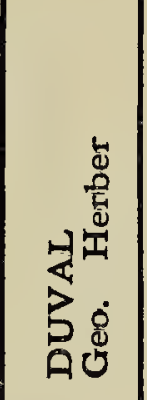 & 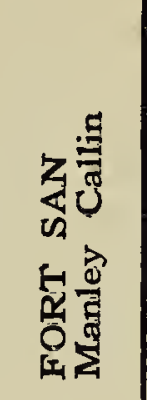 & 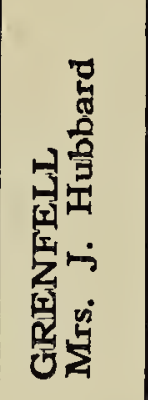 & 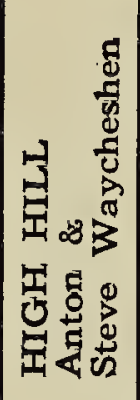 & 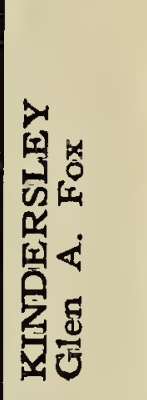 & 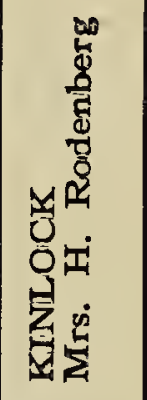 & 焉 & 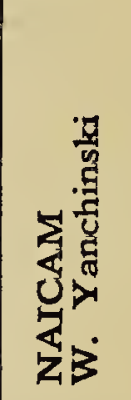 \\
\hline histling Swan & Ap 6 & Ap20 & Ap19 & & My 7 & Ap26 & & Ap13 & \\
\hline anada Goose & Ap 8 & Mr30 & Mr31 & & Ap 1 & Mr31 & Ap 8 & Ap 5 & Ap 9 \\
\hline allard & Ap 4 & Mr31 & Ap 4 & $\operatorname{Mr} 31$ & Ap 4 & Ap 1 & Ap2 1 & Ap 4 & Ap 6 \\
\hline 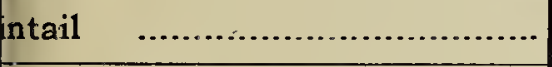 & Mr31 & Ap 1 & Ap 1 & Ap 1 & Ap 7 & Ap 1 & Ap1 1 & Ap 4 & Ap 5 \\
\hline Iarsh Hawk ...... & Mr30 & Ap 1 & $\operatorname{Mr} 28$ & Mr30 & Ap 9 & $\operatorname{Mr} 31$ & Ap10 & $\mathrm{Mr} 30$ & Mr29 \\
\hline illdeer & Mr30 & Ap 2 & Mr23 & Ap 1 & Ap 4 & Ap 2 & Ap 2 & Ap 7 & Ap 7 \\
\hline ommon Snipe & & Ap28 & Ap27 & & Ap11 & Ap19 & Ap25 & Ap26 & \\
\hline lourning Dove & My12 & Ap30 & Ap2.1 & My 14 & My 5 & My 16 & My 8 & My 4 & My 13 \\
\hline bmmon Nighthawk & My28 & & My25 & & My24 & Jn 2 & My10 & My25 & \\
\hline uby-throated Hummingbird ... & & & My19 & & & & My 15 & Jn 9 & \\
\hline ellow-Shafted Flicker & Ap18 & Ap16 & Ap13 & Ap13 & Ap15 & Ap26 & Ap24 & Ap 9 & Ap22 \\
\hline astern Kingbird ...................... & My13 & My 18 & My11 & My13 & My15 & My17 & & My 19 & My 18 \\
\hline astern Phoebe $\quad . . . . . . . . . . . . . . . .$. & & & Ap2 7 & My25 & Ap20 & My 4 & Ap29 & Ap17 & Ap24 \\
\hline arn Swallow & My 4 & My 6 & My 2 & Ap26 & My 8 & My 18 & My 8 & My 6 & My 6 \\
\hline Irple Martin ................ & & My12 & Ap2 7 & My 1 & My13 & Ap25 & Ap28 & $\mathrm{Jn} 3$ & My 4 \\
\hline 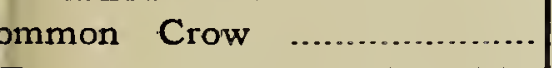 & Mr28 & Mr29 & Mr25 & Mr27 & Mr28 & Mr31 & $\operatorname{Mr} 27$ & Mr26 & Mr28 \\
\hline 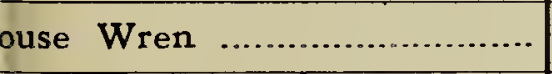 & My 21 & My 15 & My 5 & My 14 & My15 & $\mathrm{Jn} 9$ & My11 & My 11 & My 16 \\
\hline ................................... & My22 & Ap 7 & My 20 & My23 & My27 & Jn 2 & & My28 & My25 \\
\hline own Thrasher ....................... & My15 & & My10 & My20 & & My19 & & & \\
\hline ed-eyed Vireo ........................... & & & My25 & & & My23 & & My26 & My 15 \\
\hline 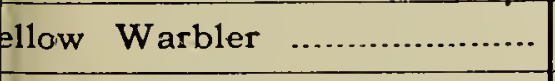 & My20 & My23 & My10 & My 13 & My23 & Mỳ10 & My 18 & My12 & My 17 \\
\hline 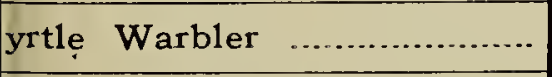 & & & My 2 & My25 & Ap22 & My 4 & My 14 & Ap30 & \\
\hline edwinged Blackbird ............... & Ap 6 & Mr28 & Ap 3 & Ap 2 & Mr30 & Ap 2 & Ap 8 & Ap 6 & Ap 6 \\
\hline ltimore Oriole $\quad \ldots \ldots \ldots \ldots \ldots \ldots \ldots$ & My19. & My24 & My 15 & My 19 & My29 & My25 & My18 & My23 & My23 \\
\hline Dse-breasted Grosbeak ........ & & & My25 & & My23 & & Ap26 & & \\
\hline merican Goldfinch ................. & My2 1 & My28 & My22 & My22 & My23 & My 4 & My21 & My25 & My24 \\
\hline ate-colored Junco .................... & Mr27 & Mr26 & Mr27 & Mr30 & Mr27 & Ap 4 & Mr27 & Mr30 & Mr31 \\
\hline hipping Sparrow $\quad \ldots \ldots \ldots \ldots \ldots \ldots . . . . . . .$. & My18 & Ap 2 & My 5 & My 5 & & My 10 & Ap25 & Ap18 & My18 \\
\hline hite-crowned Sparrow ........... & & & & My 9 & & My 4 & Ap30 & My 9 & My 9 \\
\hline hite-throated Sparrow ........ & My25 & Ap24 & My 6 & My 6 & My 6 & My 3 & Ap30 & My 5 & My 10 \\
\hline
\end{tabular}




\section{Co-operative $\mathrm{S}{ }^{\mathrm{i}}$}

Compiled by Mary Houston, Yorkton.

\begin{tabular}{|c|c|c|c|c|c|c|c|c|c|}
\hline & 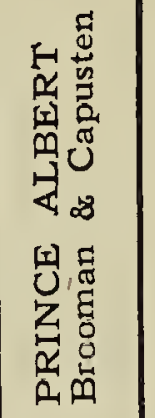 & 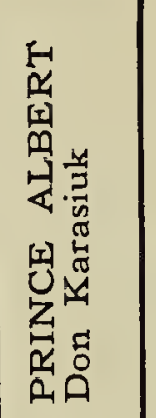 & 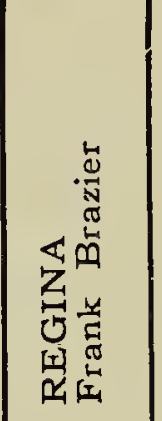 & 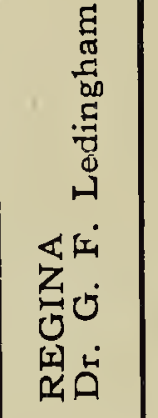 & 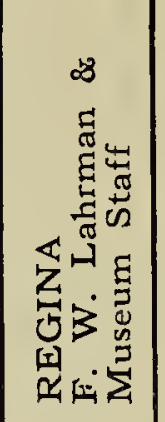 & 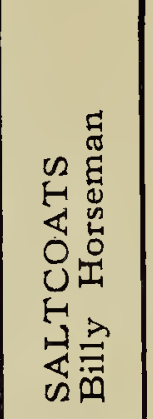 & 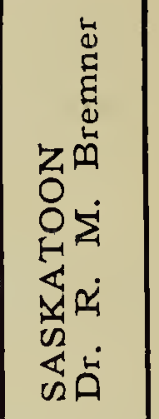 & 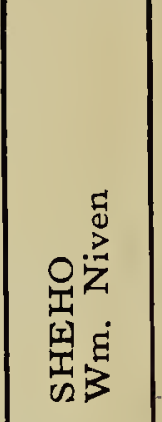 & 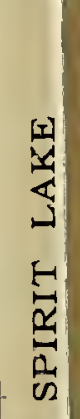 \\
\hline Whistling Swan ...... & My 2 & & Mr29 & Mr29 & Mr29 & Ap15 & Ap 12 & Ap20 & \\
\hline Canada Goose ...... & & & & Ap 6 & Mr30 & Mr31 & Ap 12 & Mr31 & $\mathrm{A}_{1}$ \\
\hline Mallard ............... & Ap 4 & Ap:10 & Mr27 & Mr28 & Mr26 & $\operatorname{Mr} 29$ & Ap 5 & Ap 2 & $\mathbf{M}$ \\
\hline Pintail & & Ap10 & $\operatorname{Mr} 28$ & Mr25 & Mr25 & $\operatorname{Mr} 30$ & Ap 6 & Ap 3 & $\mathbf{M}$ \\
\hline Marsh Hawk ....... & Ap20 & Ap 7 & Ap 4 & Ap 6 & $\operatorname{Mr} 29$ & $\operatorname{Mr23}$ & Ap 3 & Mr25 & $\mathbf{M}$ \\
\hline Killdeer & $\operatorname{Mr26}$ & $\operatorname{Mr29}$ & $\operatorname{Mr} 30$ & Ap 1 & Mr30 & Ap 1 & Ap 8 & $\operatorname{Mr} 31$ & $\mathbf{M}$ \\
\hline Common Snipe ..... & & & Ap 19 & Ap25 & Ap11 & Ap13 & Ap27 & & $\mathrm{Al}_{\mathrm{F}}$ \\
\hline Mourning Dove & & My 7 & Ap30 & Ap2 1 & Ap13 & Ap2 7 & My4 & My 2 & $\mathbf{M}$ \\
\hline Common Nighthawk & My 25 & My24 & My20 & My 15 & My 25 & My 18 & My21 & My23 & $\mathbf{M}$ \\
\hline Ruby-throated Hummingbird .. & My26 & My28 & & & & $\mathrm{Jn} 10$ & & & \\
\hline Yellow-Shafted Flicker & Ap26 & Ap25 & Ap 15 & Ap10 & Ap10 & Ap10 & Ap13 & Ap 8 & $A_{F}$ \\
\hline Eastern Kingbird ...... & My25 & My25 & My 15 & My 11 & My 12 & My 14 & My23 & My 15 & $\mathbf{M}$ \\
\hline Eastern Phoebe & My24 & My21 & & Ap2 1 & & Ap29 & & Ap16 & $\mathrm{AF}$ \\
\hline Barn Swallow ......... & & My22 & Ap27 & My 10 & Ap12 & My 4 & My25 & My 7 & $\mathbf{M}$ \\
\hline Purple Martin ........ & My20 & My 27 & My 17 & My 2 & Ap29 & Ap25 & & & M: \\
\hline Common Crow & $\operatorname{Mr} 30$ & $\mathrm{Fb} 23$ & $\operatorname{Mr29}$ & $\operatorname{Mr} 29$ & $\operatorname{Mr} 23$ & Mr21 & $\operatorname{Mr} 30$ & $\mathrm{Mr} 28$ & $\mathbf{M}$ \\
\hline House Wren ...................... & My22 & My 15 & My 11 & My 15 & My 18 & My 12 & My 7 & My 13 & $\mathrm{M}$ \\
\hline Catbird & My25 & My 25 & My24 & My22 & My23 & My23 & My25 & My22 & $\mathrm{M}:$ \\
\hline Brown Thrasher & & My21 & My 18 & My 9 & My 16 & My 12 & My 14 & My 13 & $\mathrm{M}$ \\
\hline Red-eyed Vireo $\ldots . . \ldots \ldots \ldots \ldots \ldots . . . . . . . .$. & My25 & & My24 & My 24 & My 19 & & & My 14 & $\mathrm{Mj}$ \\
\hline Yellow Warbler ....................... & My21 & & My 8 & My 9 & My 6 & My 10 & My 13 & My 13 & $\mathrm{Mj}$ \\
\hline Myrtle Warbler ......................... & & & Ap 10 & Ap19 & My 2 & Ap2.6 & & Ap29 & Ap \\
\hline Redwinged Blackbird .............. & Ap 4 & My20 & Mr30 & Ap 1 & Mr30 & Mr26 & Ap 6 & Mr31. & $\mathrm{Ap}$ \\
\hline Baltimore Oriole $\quad . . . . . . . . . . . . . . .$. & My25 & My24 & My 15 & My 15 & My 14 & My 17 & My 18 & My 16 & My \\
\hline Rose-breasted Grosbeak ........ & My25 & My25 & My24 & My 16 & My19 & & & & My \\
\hline American Goldfinch .................. & & $\mathrm{Jn} 12$ & My 11 & My24 & My 17 & My20 & My 24 & My2 1 & $\mathrm{MJ}_{3}$ \\
\hline Slate-colored Junco .................. & & Mr29 & $\operatorname{Mr} 26$ & $\operatorname{Mr} 27$ & Mr26 & $\operatorname{Mr} 26$ & Mr30 & $\operatorname{Mr} 28$ & $\mathrm{Mr}_{\mathbf{r}}$ \\
\hline Chipping Sparrow $\ldots \ldots \ldots \ldots \ldots \ldots$ & & My 1 & My 2 & My 3 & My 9 & Ap20 & My 6 & My 2 & Mu \\
\hline White-crowned Sparrow .......... & & My 8 & My 2 & My 2 & My 2 & Ap29 & & & $\mathrm{My}$ \\
\hline White-throated Sparrow ........ & My23 & My 4 & My 2 & My 2 & My 3 & Ap2 7 & My 4 & My 2 & My \\
\hline
\end{tabular}




\section{ration Study 1958}

\begin{tabular}{|c|c|c|c|c|c|c|c|c|}
\hline & 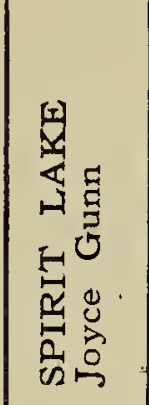 & 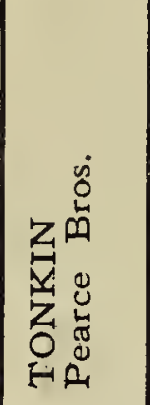 & 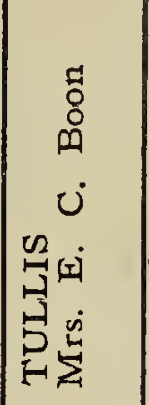 & 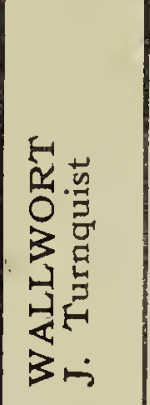 & 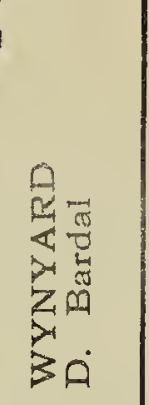 & 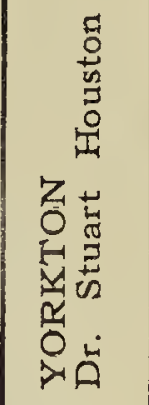 & 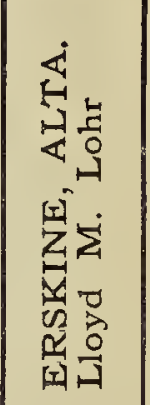 & 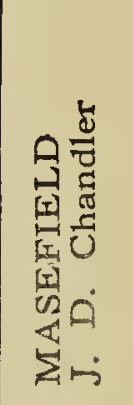 \\
\hline Whistling Swan & My 3 & & Ap19 & & & Ap20 & Ap 15 & \\
\hline Canada Goose …............................. & Ap11 & Ap 8 & Ap11 & ' & Mr27 & & Ap 6 & Mr30 \\
\hline Mallard ...... & Ap 7 & Mr31 & Ap 6 & & $\mathrm{M} 27$ & Ap 4 & Ap 4 & Ap 3 \\
\hline Pintail & Mr31 & Ap 7 & Mr29 & & Ap 3 & Ap 4 & Ap 5 & Mr26 \\
\hline 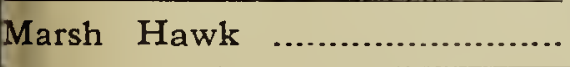 & Mr30 & Ap 7 & Ap13 & & Ap12 & Ap 6 & Ap 13 & Mr20 \\
\hline Killdeer $\quad$.............. & Ap 1 & Mr31 & Ap 4 & & Ap 7 & $\operatorname{Mr} 31$ & & Mr30 \\
\hline Common Snipe & Ap12 & Ap2 6 & & & & Ap20 & & \\
\hline Mourning Dove ........................... & Ap23 & My 10 & & My 11 & My 3 & My11 & Jn 3 & My 5 \\
\hline Common Nighthawk & My23 & My23 & & & My 3 & & & \\
\hline Ruby-throated Hummingbird . & My26 & My21 & & My20 & My31 & My25 & & \\
\hline Yellow-Shafted Flicker & Ap16 & Ap 8 & & & Ap18 & Ap20 & Ap20 & \\
\hline Eastern Kingbird …...................... & My13 & My 14 & My22 & My20 & My19 & My 18 & My 13 & My18 \\
\hline Eastern Phoebe …................... & Ap10 & & & My 9 & & My18 & My13 & Ap27 \\
\hline Barn Swallow ..... & My 5 & Ap2 7 & My 12 & My 12 & My24 & My4 & My 9 & My 5 \\
\hline Purple Martin …........................ & My 13 & & & My 10 & & My 2 & Ap18 & \\
\hline Common Crow …............ & $\operatorname{Mr} 27$ & & Mr29 & & Mr27 & $\operatorname{Mr26}$ & $\operatorname{Mr} 28$ & Mr22 \\
\hline House Wren & My 9 & My 10 & & My23 & My 14 & My13 & My 14 & \\
\hline Catbird $\quad \cdots \ldots \ldots \ldots \ldots \ldots \ldots \ldots \ldots \ldots . . . \cdots \cdots$ & My 18 & My 8 & Jn 1 & & My25 & My25 & & \\
\hline Brown Thrasher ........... & My 15 & & & & My21 & My25 & Jn 2 & \\
\hline \multicolumn{9}{|l|}{ Red-eyed Vireo ............................ } \\
\hline 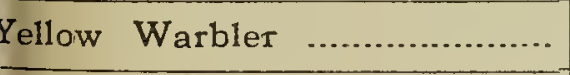 & My14 & My 16 & & & My21 & My13 & My10 & \\
\hline Myrtle Warbler & Ap2 8 & & & & & My11 & & \\
\hline Redwinged Blackbird ….............. & Mr31 & Ap 7 & My12 & & Ap20 & Ap 4 & Ap 6 & Ap 6 \\
\hline 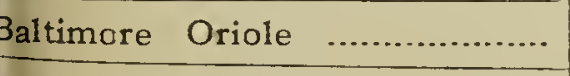 & My15 & My 15 & & & My 17 & My 18 & $\mathrm{My}_{\mathrm{y}} 13$ & My26 \\
\hline Rose-breasted Grosbeak ......... & & $\mathrm{My} 25$ & & & & $\dot{M} y 18$ & & \\
\hline 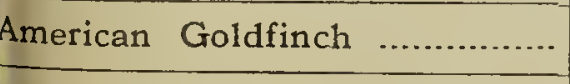 & $\mathrm{Jn} 2$ & & & & My30 & My25 & My26 & \\
\hline Slate-colored Junco …….............. & Mr26 & Ap 3 & Ap 6 & & Mr26 & Ap 4 & $\mathrm{Ap} 7$ & \\
\hline Chipping Sparrow ………….... & Ap29 & & & & My18 & & & \\
\hline White-crowned Sparrow ................ & & & My 4 & & My 7 & My 10 & Jn 8 & \\
\hline White-throated Sparrow ............. & My 4 & & & & My 7 & My 2 & & \\
\hline
\end{tabular}

